\title{
CPW Fed T-Shaped Wearable Antenna for ISM Band, Wi-Fi, WiMAX, WLAN and Fixed Satellite Service Applications
}

\author{
Mudunuri Padmanabha Raju • D. S. Phani Kishore • B. T. P. Madhav"
}

\begin{abstract}
The placement of wearable products integrated with telecommunication systems is having a good impact on healthcare communication all over the world. Embedded communication devices like antennas and RF devices are helping doctors to assess the condition of patients from remote locations. The present work deals with the design and development of a coplanar waveguide fed wearable antenna for ISM band (2.4-2.5 GHz), Wi-Fi, WLAN (2.4-2.48 GHz), WiMAX (3.4-3.6 GHz), and fixed satellite service (3.6-3.7 GHz and 9-11.5 $\mathrm{GHz})$ applications. The designed antenna on jeans substrate $(50 \times 40 \times 1.6 \mathrm{~mm})$ provides a $1.7 \mathrm{GHz}$ bandwidth with a gain of $4.6 \mathrm{~dB}$. A parametric study of different fabrics and the performance characteristics of bending angles are also provided in this work from the application point of view. The simulated results obtained from CST tool and measured results of fabric material based antenna on vector network analyzer are correlated with each other.
\end{abstract}

Key Words: Coplanar Waveguide, Industrial Scientific and Medical Band (ISM), Wearable Antenna, Wireless Applications.

\section{INTRODUCTION}

The design of wearable devices opens the doors to mechanisms for monitoring a human being's health condition. Intelligent wearable devices embedded in textile materials will help considerably with extracting certain parameters of the body to monitor its condition. Medical monitoring is very essential in certain conditions for people who are involved in mining, military, security operations and other risky environments. These people's health-related data should be transmitted to hospitals and doctors to enable medical assistance in critical situations. Hence, for all these broad applications of data transmission, wearable antennas are an optimal solution $[1,2]$.
The wireless body area networks associated with wearable antennas should provide solutions for different applications with low transmission power, less complexity, and minimum interference [3-6]. A microstrip patch structure can provide a solution for the design of such wearable antennas in conformal and flexible nature with placement in the clothing $[7,8]$. Recently people having been trying to design antennas with textile materials with conductive coating and, in some cases, with 100\% pure textiles only $[9,10]$. A patient-friendly model can be designed with a washable fabric-based antenna system.

Unlike previous wearable antennas $[11,12]$, the present antenna design is found to be capable of meeting the requirements of wearable electronic devices, such as being robust, consuming

Manuscript received September 3, 2018 ; Revised November 17, 2018 ; Accepted February 28, 2019. (ID No. 20180903-063J)

Department of Electronics \& Communication Engineering, Koneru Lakshmaiah Education Foundation, Andhra Pradesh, India.

"Corresponding Author: B. T. P. Madhav (e-mail: btpmadhav@kluniversity.in)

This is an Open-Access article distributed under the terms of the Creative Commons Attribution Non-Commercial License (http://creativecommons.org/licenses/by-nc/4.0) which permits unrestricted non-commercial use, distribution, and reproduction in any medium, provided the original work is properly cited.

(c) Copyright The Korean Institute of Electromagnetic Engineering and Science. All Rights Reserved. 
a small amount of power, comfortable to wear with flexible materials. In addition, the current manuscript materials used can guarantee washability of the wearable device and, accordingly, its reuse. The measured results of the present antenna designs are compared with simulations, and good agreement is observed.

\section{ANTENNA GEOMETRY}

A T-shaped coplanar waveguide fed antenna is designed on a fabric material-based substrate. The proposed model is presented in Fig. 1; it occupies the dimensions of $50 \times 42 \times 0.6 \mathrm{~mm}$ (Table 1). The antenna is constructed based on the coplanar waveguide structure using the design equations.

The characteristic impedance can be calculated from

$$
Z_{0}=\frac{60 \pi}{\sqrt{\varepsilon_{\text {eff }}}} \frac{1}{\frac{K(k)}{K\left(k^{\prime}\right)}+\frac{K(k l)}{K\left(k l^{\prime}\right)}}
$$

Here $k^{\prime}$ and $l^{\prime}$ are the complementary modules of $K$ and 1 . where

$$
\begin{gathered}
k=W_{f} / g \\
g=W f+2 g
\end{gathered}
$$

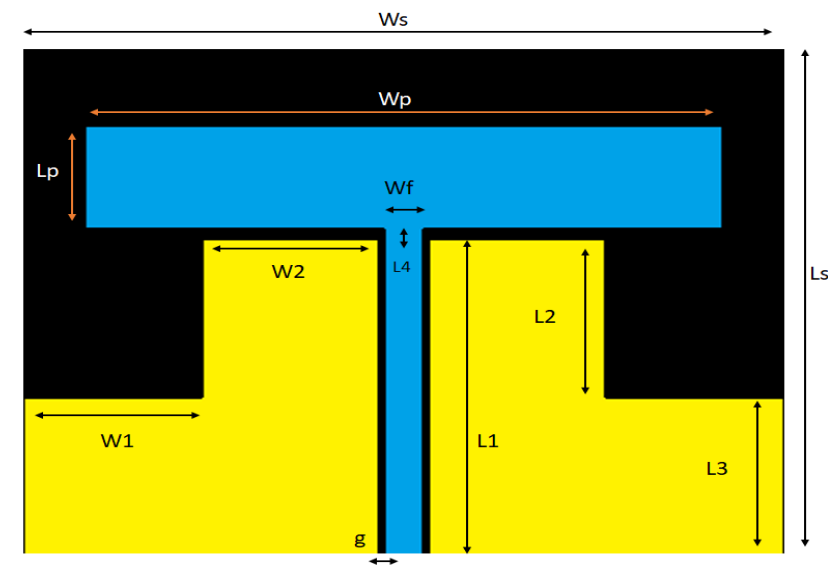

(a)

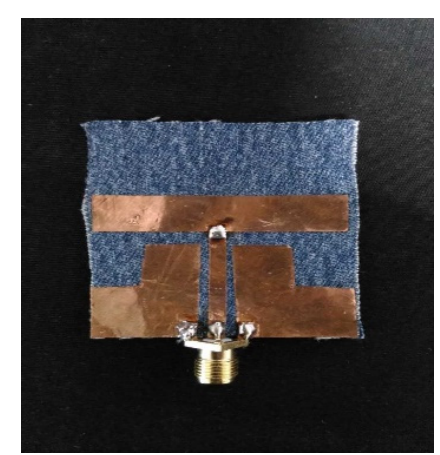

(b)

Fig. 1. Coplanar waveguide fed T-shaped antenna: (a) simulated model and (b) fabricated model.
Table 1. Antenna dimensions

\begin{tabular}{lccc}
\hline Parameter & $\begin{array}{c}\text { Dimension } \\
(\mathrm{mm})\end{array}$ & Parameter & $\begin{array}{c}\text { Dimension } \\
(\mathrm{mm})\end{array}$ \\
\hline$W_{s}$ & 50 & $L_{s}$ & 40 \\
$W_{p}$ & 42 & $L_{p}$ & 8 \\
$W_{1}$ & 12 & $L_{1}$ & 24 \\
$W_{2}$ & 11 & $L_{2}$ & 12 \\
$W_{f}$ & 2 & $L_{3}$ & 12 \\
$g$ & 0.5 & $L_{4}$ & 1 \\
\hline
\end{tabular}

$W_{f}$ is the width of the feed line and $g$ is the gap between the feed line and the ground plane.

$$
\begin{gathered}
k^{\prime}=\sqrt{1-k^{2}} \\
k l^{\prime}=\sqrt{1-k l^{2}} \\
k l=\frac{\tanh \left(\frac{\pi w_{f}}{4 h}\right)}{\tanh \left(\frac{\pi b}{4 h}\right)}
\end{gathered}
$$

where $b$ is the height of the substrate and $\varepsilon_{e f f}$ is the effective dielectric constant of the material.

$$
\varepsilon_{e f f}=\frac{1+\varepsilon_{r} \frac{K\left(k^{\prime}\right)}{K(k)}+\frac{K(k l)}{K\left(k l^{\prime}\right)}}{1+\frac{K\left(k^{\prime}\right)}{K(k)}+\frac{K(k l)}{K\left(k l^{\prime}\right)}} .
$$

\section{RESULTS AND DISCUSSION}

The designed model is simulated using a CST Microwave Studio and the corresponding results are presented in this section. Fig. 2 shows the reflection coefficient of the antenna with respect to the frequency. The antenna operates at two bands of 2-4 GHz and 9-11.5 GHz with a bandwidth of $2 \mathrm{GHz}$ at fundamental resonant frequency and $1.5 \mathrm{GHz}$ at second resonant frequency, respectively.

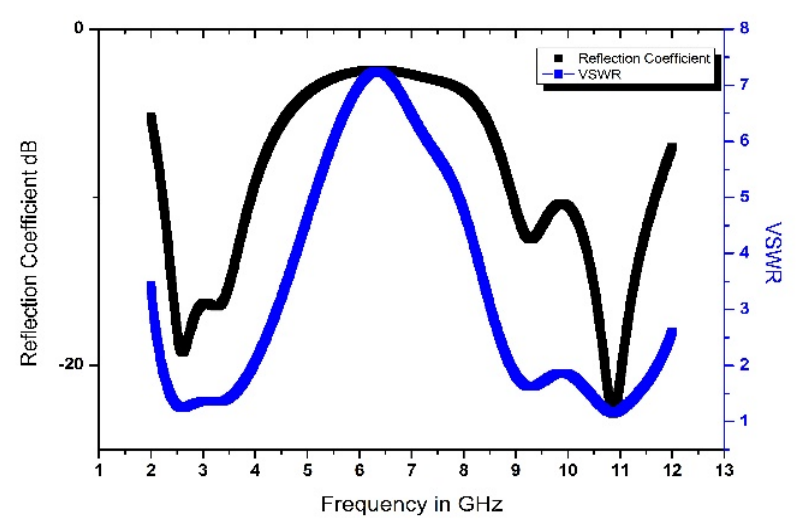

Fig. 2. Reflection coefficient vs. frequency. 
A parametric analysis of the antenna with respect to its dimensional characteristics was performed. The length of the patch is varies from $6 \mathrm{~mm}$ to $8 \mathrm{~mm}$ and the corresponding reflection coefficient is presented in Fig. 3. The bandwidth reduced when the length of the patch is decreased from 8 to 6 $\mathrm{mm}$. The dimension of $8 \mathrm{~mm}$ for $L p$ is fixed after optimized results are obtained. The feed width $(W f)$ is varies from 2 to 3 $\mathrm{mm}$ and the corresponding results with the reflection coefficient presented in Fig. 4.

Similarly, the width of the patch $(W p)$ is varies from 36 to 42 $\mathrm{mm}$ and is fixed at $42 \mathrm{~mm}$ after optimized results were obtained for this dimension (Fig. 5).

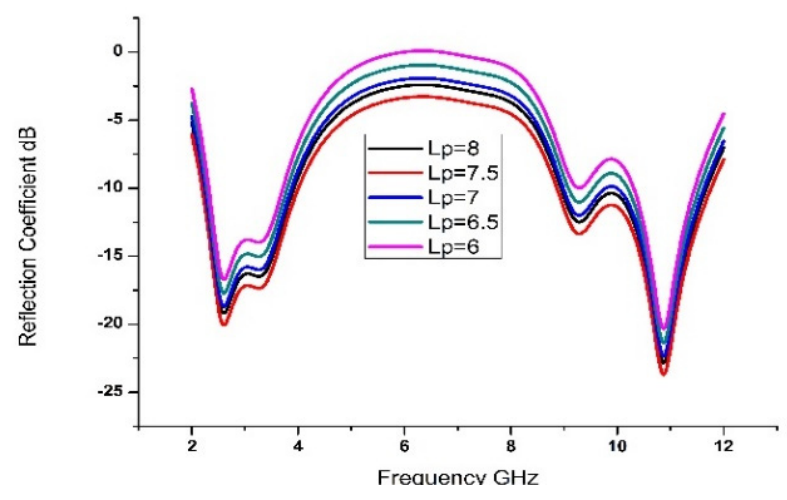

Fig. 3. Parametric analysis with change in length of the patch, $L_{p}$.

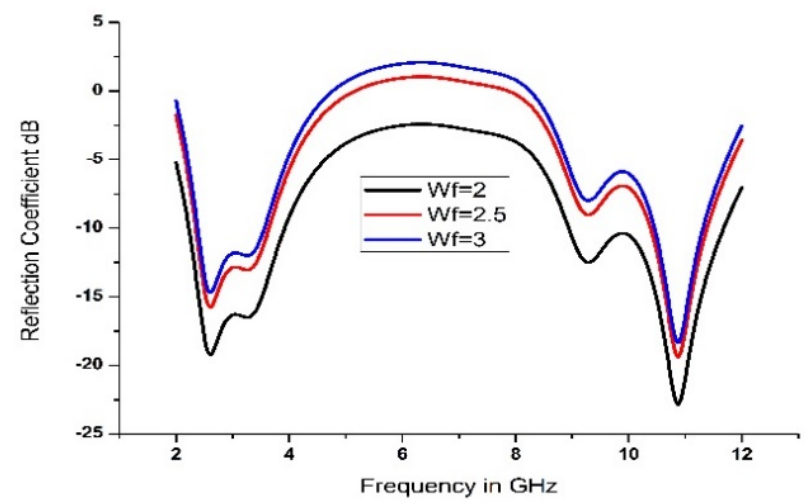

Fig. 4. Parametric analysis with change in feed width, $W_{f}$.

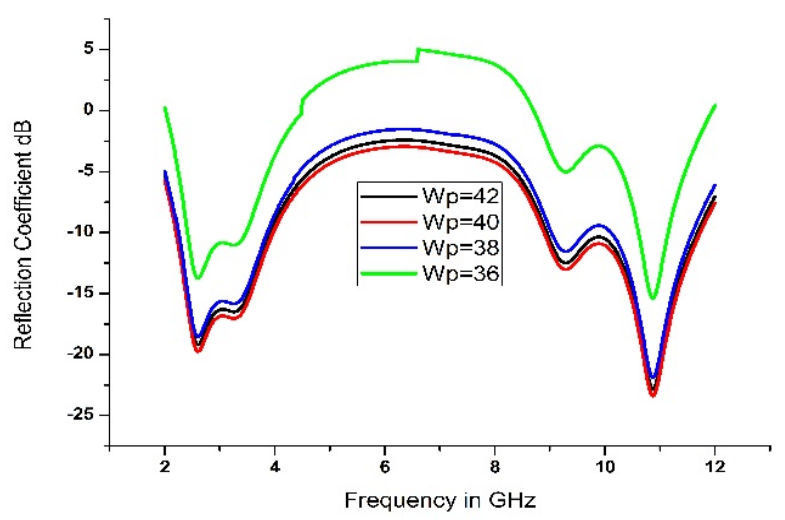

Fig. 5. Parametric analysis with change in feed width, $W_{p}$.
The radiation characteristics of the antenna are presented in Fig. 6 for two different frequencies, $2.5 \mathrm{GHz}$ and $3.6 \mathrm{GHz}$ in E-plane and H-plane. In H-plane, an omnidirectional pattern can be observed.

The simulated surface current distribution of the antenna at three operating frequencies presented in Fig. 7. The current distribution is more at the feed line and on the lower part of the antenna at three resonant frequencies. The upper edges of the ground plane are also showing some significance distribution from the obtained results.

A time domain analysis of the antenna is presented in Fig. 8. The input signal and the corresponding impulse response can be
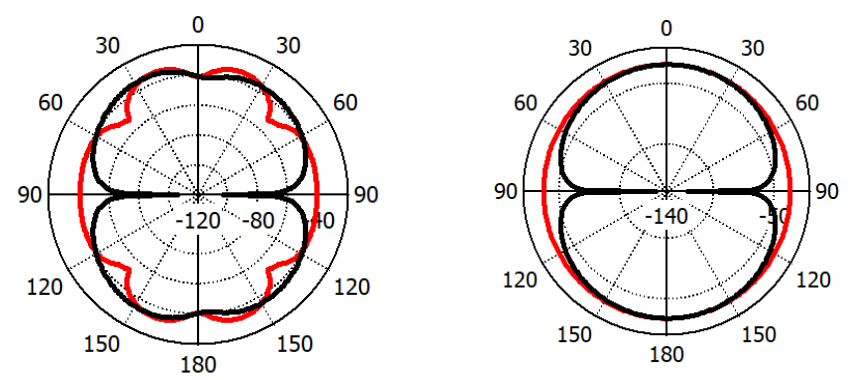

(a)
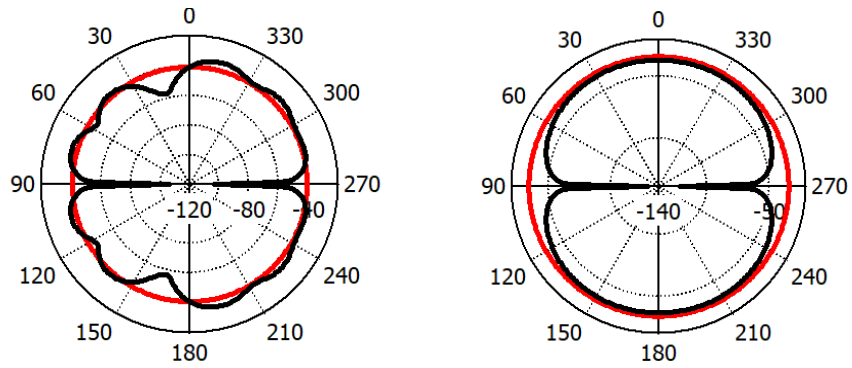

(b)

Fig. 6. Measured radiation pattern of the antenna: (a) $2.5 \mathrm{GHz}$ and (b) 3.6 GHz.

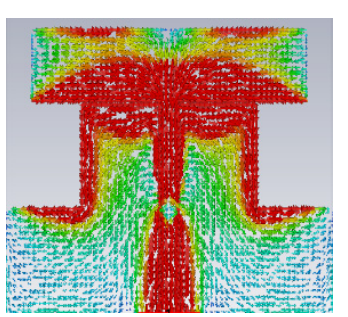

(a)

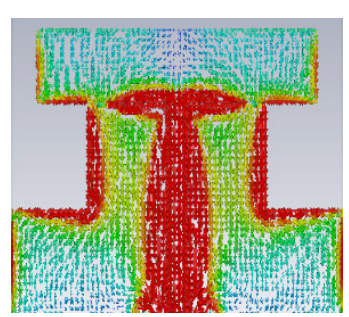

(b)

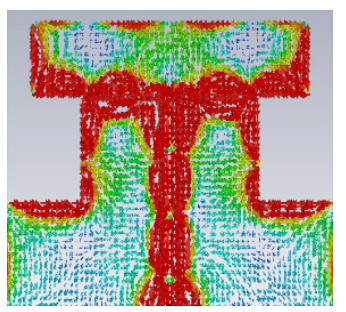

(c)

Fig. 7. Surface current distribution: (a) $2.5 \mathrm{GHz}$, (b) $3.6 \mathrm{GHz}$, and (c) $11 \mathrm{GHz}$. 


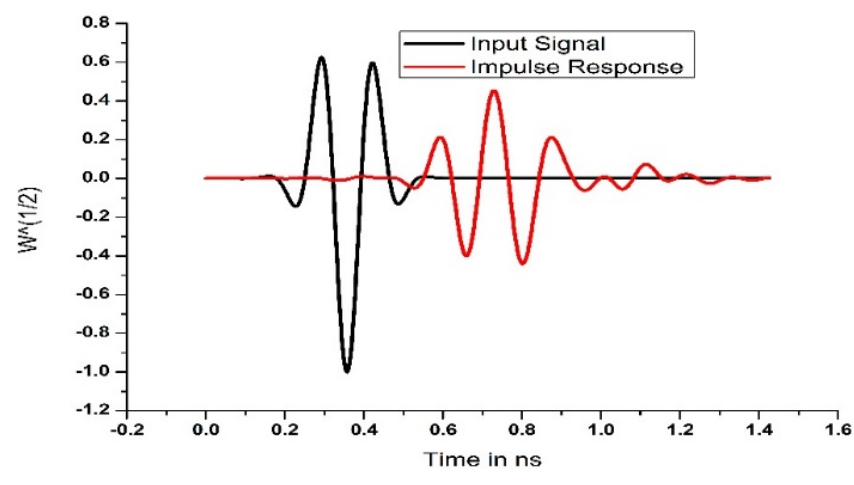

Fig. 8. Time domain analysis of the proposed antenna.

observed from the result. A small distortion in the response is due to a mismatch in timing. Fig. 9 shows the testing of the antenna with an Anritsu combinational analyzer.

\section{STUdy OF OTher TeXtile Substrate MATERIALS}

This work is extended to encompass a study of the antenna performance characteristics of other textile substrate materials. A list of materials selected for the study is presented in Table 2.

The reflection coefficient and the gain plots for the proposed antenna of different textile materials are analyzed and presented in Figs. 10 and 11, respectively. It can be observed that the antenna impedance bandwidth changes for each fabric from Fig. 10. There is a shift in the operating frequencies of the antenna with respect to the change in the fabric material. A peak realized gain of $4.5 \mathrm{~dB}$ is attained for jeans cotton and $4.2 \mathrm{~dB}$ for polyester.

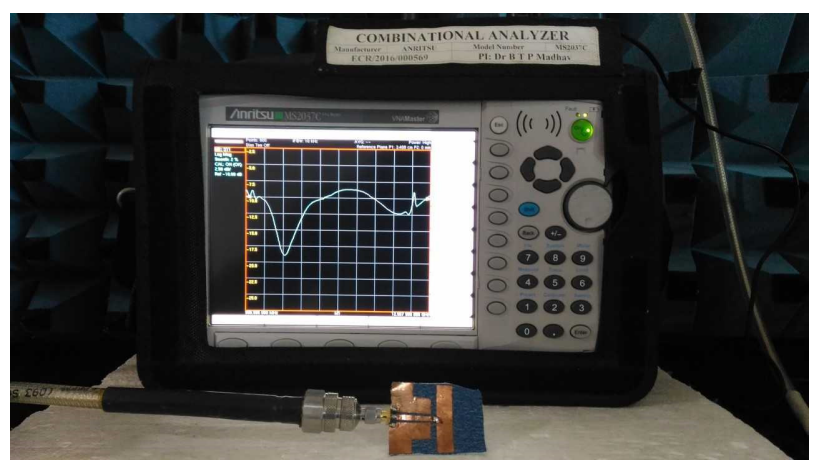

Fig. 9. Measurement on Anritsu combinational analyzer.

Table 2. Textile materials parameters

\begin{tabular}{lcc}
\hline $\begin{array}{c}\text { Textile substrate } \\
\text { material }\end{array}$ & Dielectric constant & $\begin{array}{c}\text { Effective dielectric } \\
\text { constant }\end{array}$ \\
\hline Polycot & 1.3 & 1.8 \\
Polyester & 1.4 & 1.39 \\
Jeans cotton & 1.6 & 1.59 \\
Wash cotton & 1.61 & 1.45 \\
Bed sheet & 1.8 & 1.4 \\
\hline
\end{tabular}

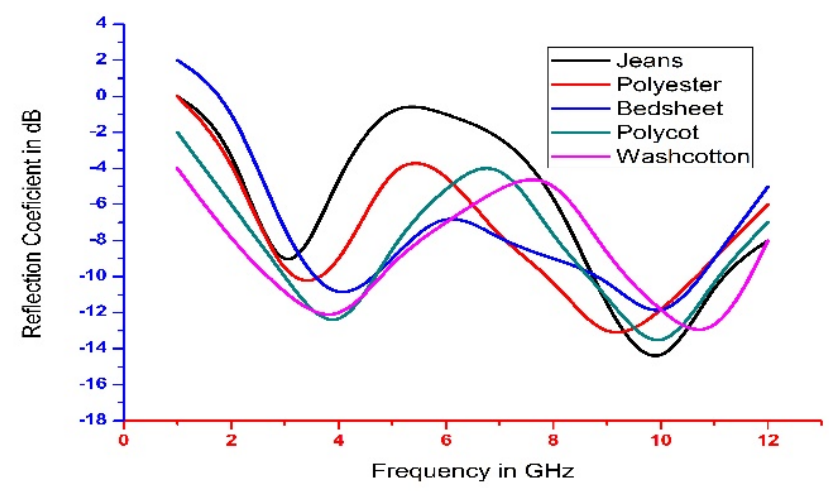

Fig. 10. Reflection coefficient vs. frequency for different substrate materials.

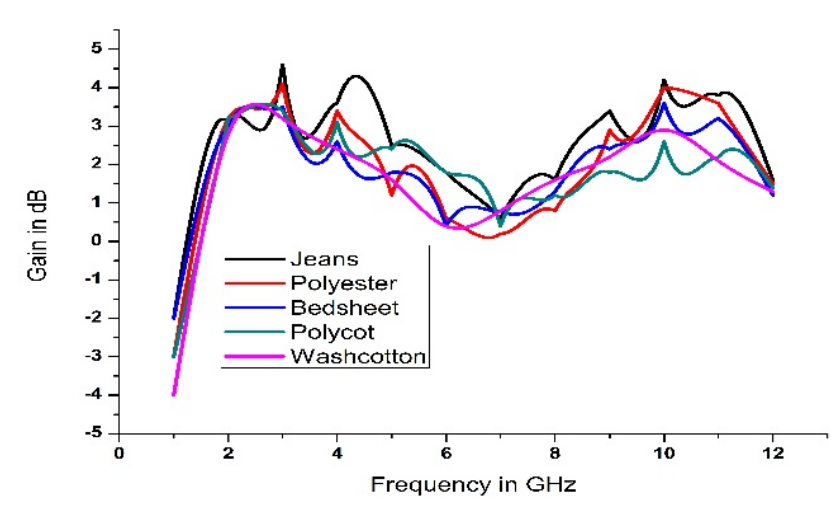

Fig. 11. Gain vs. frequency for different substrate materials.

\section{Conformal Testing by Placing On Body}

After we observed the performance characteristics of the antenna with different fabric materials and we placed the model on the human body. Fig. 12 shows the placement of the antenna on the hand and on the chest and the electric field distribution around and inside at $2.5 \mathrm{GHz}$. From Fig. 12(b) we can observe that the electric field levels at the back of the body are very low and the radiation efficiency is $65 \%$. Except at the transmitting antenna, many ripples are generated all over the body.

The conformal behavior of the antenna at different bending angles are presented in Fig. 13. Three bending angles $\left(60^{\circ}, 90^{\circ}\right.$ and $120^{\circ}$ ) were selected for the experimentation and the performance of the antenna was analyzed. The reflection coefficient of the antenna at different bending angles is presented in Fig. 14.

The reflection coefficient of the antenna with different bending angles is presented here. Except for $120^{\circ}$ bending, the remaining cases show dual-band operation. For the $120^{\circ}$ bend, a notch band occurred at $10 \mathrm{GHz}$, and in this case, it is behaving like triple-band antenna. Due to low bandwidth, the notching band can be neglected in this case. The performance characteristics are almost the same with respect to the antenna in the planar condition, except for minor bandwidth variation.

The designed antenna is placed on the human hand phantom 


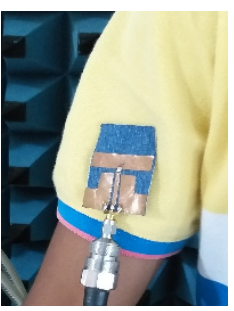

(a)

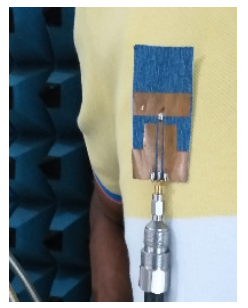

(b)

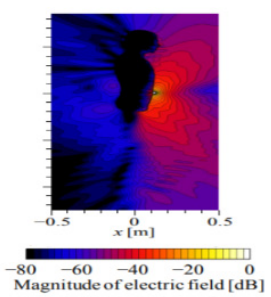

(c)
Fig. 12. Placement of the antenna and electric field distribution: (a) placement on hand, (b) placement on chest, and (c) electric field distribution.

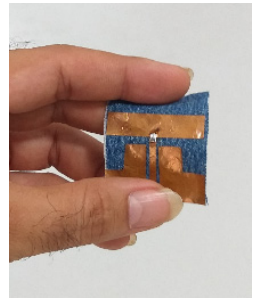

(a)

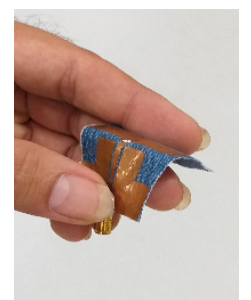

(b)

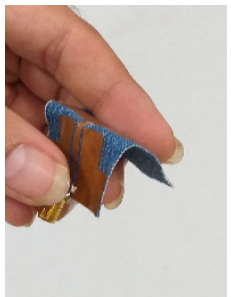

(c)
Fig. 13. Conformal testing: (a) $60^{\circ}$, (b) $90^{\circ}$, and (c) $120^{\circ}$.

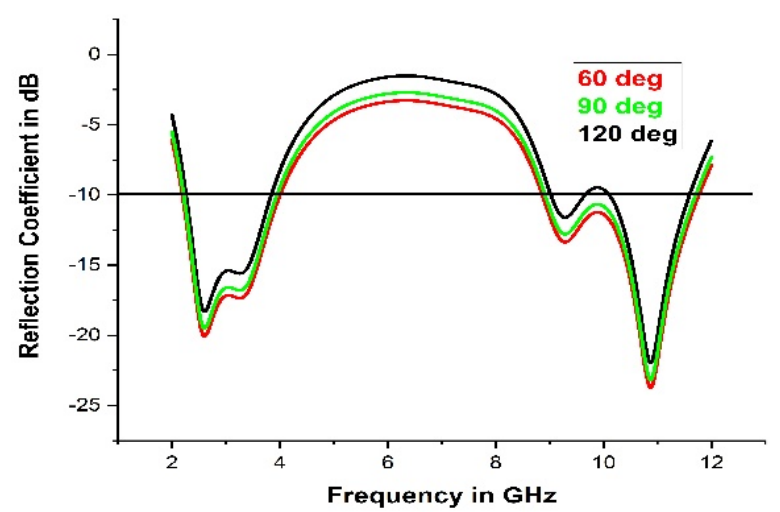

Fig. 14. Performance of the antenna with conformal behavior.

model as shown in Fig. 15(a) and a radiation analysis of the model is performed at different bending angles of $60^{\circ}, 90^{\circ}$ and $120^{\circ}$. Fig. 15(b)-(d) show the direction of the pattern in a threedimensional view at the specified bending angles.

The radiation characteristics of the antenna in the XY, YZ and ZX planes are shown in Figs. 16-18 at three bending angles. In Fig. 16, at $60^{\circ}$ bending placement on the human body, the radiation pattern is like a dipole pattern in the $\mathrm{XY}$ plane and quasi-omnidirectional in the $\mathrm{XZ}$ plane. At $90^{\circ}$ and $120^{\circ}$, the pattern is monopole-like in the XY plane. An omnidirectional pattern in the $\mathrm{XZ}$ plane for $90^{\circ}$ bending and a quasi-omnidirectional pattern in the $\mathrm{XZ}$ plane for $120^{\circ}$ can be observed in Figs. 17 and 18.

The gain of the antenna when it is placed on the human body is calculated and presented in Fig. 19. The gain variation with

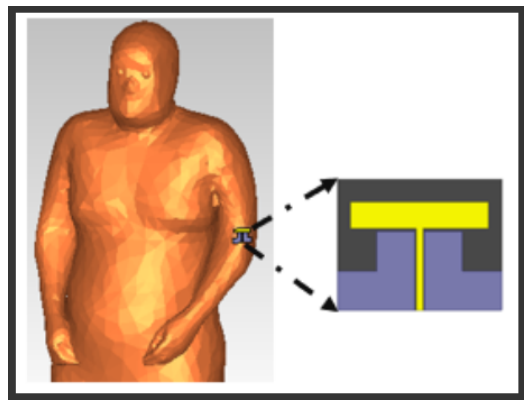

(a)

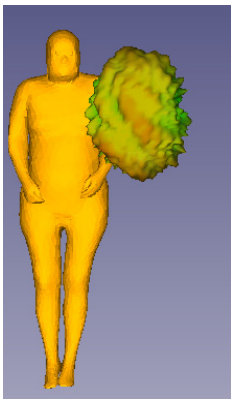

(b)

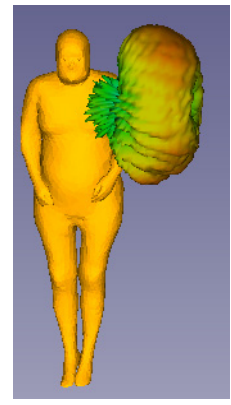

(c)

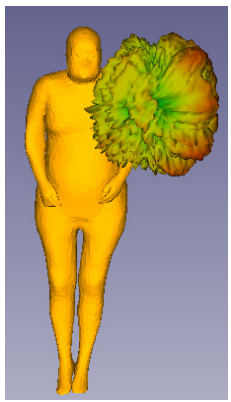

(d)
Fig. 15. Antenna placement on hand with bending angles: (a) placement on hand, (b) $60^{\circ}$, (c) $90^{\circ}$, and (d) $120^{\circ}$.

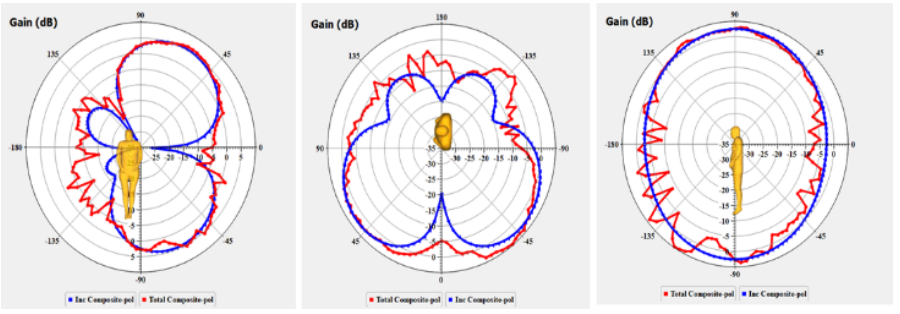

Fig. 16. Radiation pattern in $\mathrm{XY}, \mathrm{YZ}$, and $\mathrm{XZ}$ planes for bending angle of $60^{\circ}$.
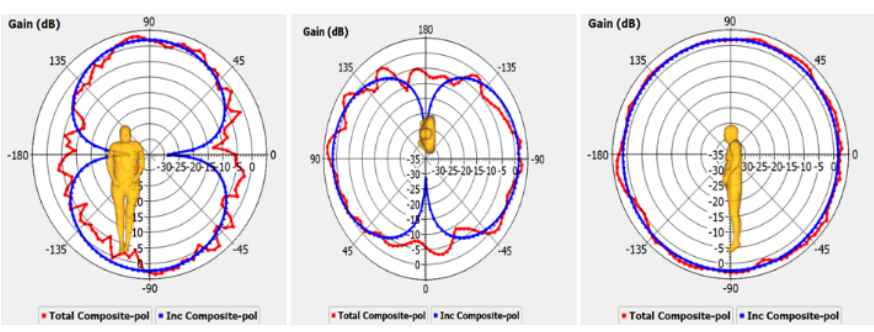

Fig. 17. Radiation pattern in $\mathrm{XY}, \mathrm{YZ}$, and $\mathrm{XZ}$ planes for bending angle of $90^{\circ}$
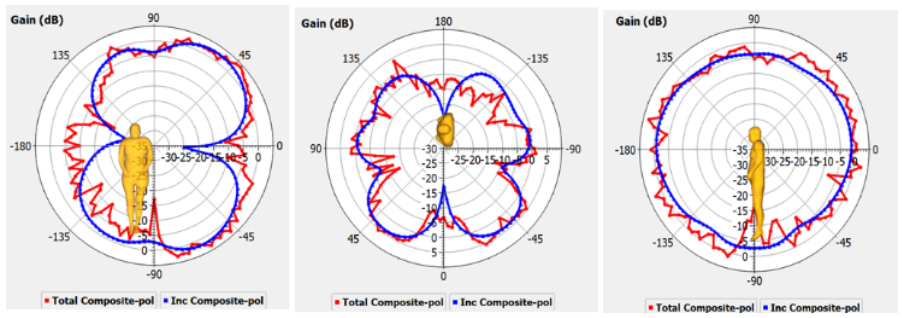

Fig. 18. Radiation pattern in $\mathrm{XY}, \mathrm{YZ}$, and $\mathrm{XZ}$ planes for bending angle of $120^{\circ}$. 


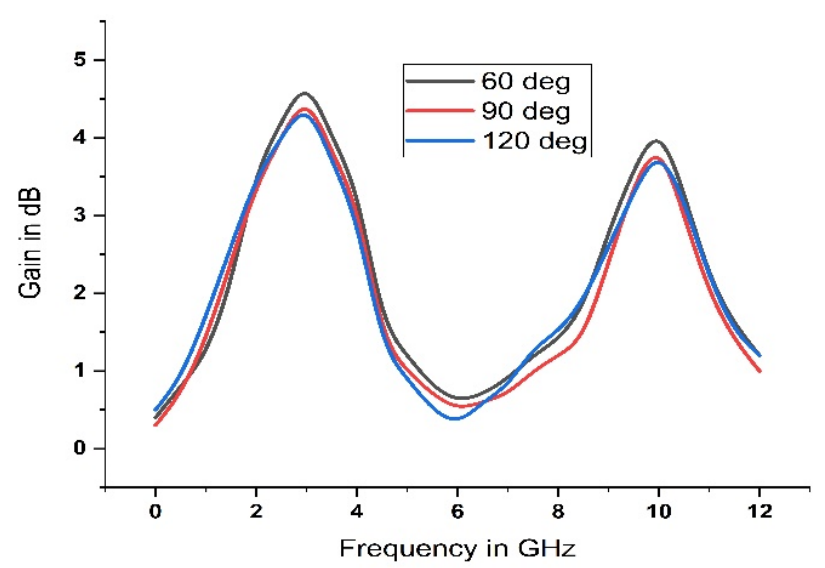

Fig. 19. Frequency vs. gain for different bending angles.

respect to the frequency of operation at the three bending angles is similar to each other, except for 0.2 to $0.3 \mathrm{~dB}$ variation.

\section{CONCLUSION}

A low-profile coplanar waveguide fed antenna is designed for ISM band (2.4-2.5 GHz), Wi-Fi, WLAN (2.4-2.48 GHz), WiMAX (3.4-3.6), fixed satellite service (3.6-3.7 GHz and 9$11.5 \mathrm{GHz}$ ) communication applications. The proposed antenna is constructed on textile substrate material of jeans cloth for wearable applications. Other fabric substrate materials like polyester, polycot, bedsheet, and wash cotton are also examined for their applicability, and their performance characteristics are presented in this work. The radiation characteristics and gain parameters with respect to the bending angles on the human body are also presented. According to these results, future antenna designers need to ensure that wearable telecommunication devices operate properly near the human body.

Our sincere thanks to the Department of Electronics \& Communication Engineering of Koneru Lakshmaiah Education Foundation and DST for their support through ECR/2016/000569 and EEQ/2016/000604.

\section{REFERENCES}

[1] S. Park and S. Jayaraman, "Enhancing the quality of life through wearable technology," IEEE Engineering in Medicine and Biology Magazine, vol. 22, no. 3, pp. 41-48, 2003.

[2] J. Tak, S. Woo, J. Kwon, and J. Choi, "Dual-band dual-mode patch antenna for on-/off-body WBAN communica- tions," IEEE Antennas and Wireless Propagation Letters, vol. 15, pp. 348-351, 2016.

[3] J. Zhao, D. Psychoudakis, C. C. Chen, and J. L. Volakis, "Design optimization of a low-profile UWB body-of-revolution monopole antenna," IEEE Transactions on Antennas and Propagation, vol. 60, no. 12, pp. 5578-5586, 2012.

[4] A. Zaric, J. R. Costa, and C. A. Fernandes, "Design and ranging performance of a low-profile UWB antenna for WBAN localization applications," IEEE Transactions on Antennas and Propagation, vol. 62, no. 12, pp. 6420-6427, 2014.

[5] M. Koohestani, J. F. Zurcher, A. A. Moreira, and A. K. Skrivervik, "A novel, low-profile, vertically-polarized UWB antenna for WBAN," IEEE Transactions on Antennas and Propagation, vol. 62, no. 4, pp. 1888-1894, 2014.

[6] W. Jeong, J. Tak, and J. Choi, "A low-profile IR-UWB antenna with ring patch for WBAN applications," IEEE Antennas and Wireless Propagation Letters, vol. 14, pp. 14471450, 2015.

[7] J. Jeon, S. Lee, J. Choi, and S. Kim, "Analysis of absorption loss by a human body in on-to-off body communication at 2.45 GHz,"Journal of Electromagnetic Engineering and Science, vol. 15, no. 2, pp. 97-103, 2015.

[8] N. Chahat, M. Zhadobov, R. Sauleau, and K. Ito, "A compact UWB antenna for on-body applications," IEEE Transactions on Antennas and Propagation, vol. 59, no. 4, pp. 11231131, 2011.

[9] J. Lee, S. I. Kwak, and S. Lim, "Wrist-wearable zeroth-order resonant antenna for wireless body area network applications," Electronics Letters, vol. 47, no. 7, pp. 431-433, 2011.

[10] W. T. Shay, S. C. Jan, and J. H. Tarng, "A reduced-size wide slot antenna for enhancing along-body radio propagation in UWB on-body communications," IEEE Transactions on Antennas and Propagation, vol. 62, no. 3, pp. 1194-1203, 2014.

[11] P. Syam Sundar, B. T. P. Madhav, D. Sri Harsha, P. Manasa, G. Manikanta, and K. Brahmaiah, "Fabric substrate material based multiband spike antenna for wearable applications," Research Journal of Applied Sciences, Engineering and Technology, vol. 8, no. 3, pp. 429-434, 2014.

[12] B. Prudhvi Nadh, B. T. P. Madhav, M. Siva Kumar, M. Venkateswara Rao, and T. Anilkumar, "Circular ring structured ultra-wideband antenna for wearable applications," International Journal of $R F$ and Microwave Computer-Aided Engineering, vol. 29, no. 4, article no. e21580, 2019. 


\section{Mudunuri Padmanabha Raju}

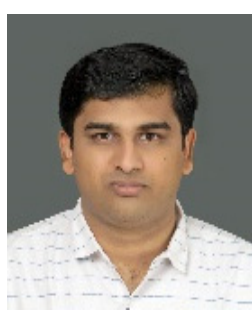

did his B.E. in Electronics and Communications at S.R.K.R. Engineering College, Bhimavaram from 2004 to 2008. He did his M.Tech. in Communication Systems at Sir C. R. Reddy Engineering College, Eluru. Presently he is pursuing a Ph.D. parttime at K. L. University, Vijayawada and working as an Assistant Professor at Shri Vishnu Engineering College for Women, Bhimavaram.

\section{S. Phani Kishore}

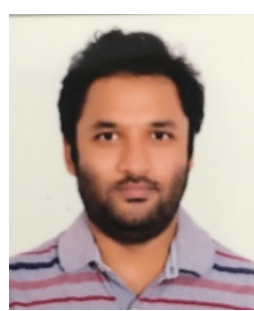

is a professor at the Department of ECE of K. L. University. He did his postdoc in Sweden and has published several papers at conferences and in journals. He is a reviewer for several peer-reviewed journals and works in the area of signal processing and communication systems. He is currently guiding four scholars towards their doctoral degrees.
B. T. P. Madhav

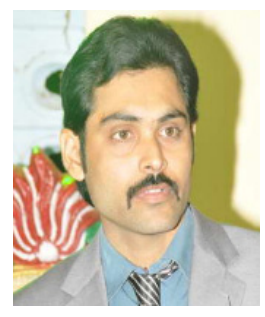

received B.Sc., M.Sc., MBA, and M.Tech. degrees from Nagarjuna University, AP, India in 2001, 2003, 2007, and 2009, respectively. He got his Ph.D. in the field of antennas from K. L. University. Currently he is a Professor and Associate Dean at K. L. University. He has published more than 328 papers in international and national journals and conferences. He is a reviewer for several international journals, including IEEE, Elsevier, Springer, Wiley, and Taylor \& Francis and has also served as a reviewer for several international conferences. His research interests include antennas, liquid crystal applications, and wireless communications. He is a member of IEEE, a life member of ISTE, IACSIT, IRACST, IAENG, and UACEE, and a fellow of IAEME. He is a member of the editorial boards of 36 journals. He has authored ten books and is guiding eight $\mathrm{Ph} . \mathrm{D}$. scholars. 